TITLE:

\title{
Molecular dynamics simulations of surface modification and damage formation by gas cluster ion impacts
}

$\operatorname{AUTHOR}(\mathrm{S}):$

Aoki, Takaaki; Matsuo, Jiro

\section{CITATION:}

Aoki, Takaaki... [et al]. Molecular dynamics simulations of surface modification and damage formation by gas cluster ion impacts. Nuclear Instruments and Methods in Physics Research Section B: Beam Interactions with Materials and Atoms 2006, 242(1-2): 517-519

ISSUE DATE:

2006-01

URL:

http://hdl.handle.net/2433/8930

\section{RIGHT:}

This is not the published version. Please cite only the published version.; この論文は出版社版でありません。引用の際には出版社版を ご確認ご利用ください。 
Molecular Dynamics Simulations of Surface Modification and Damage Formation by Gas Cluster Ion Impacts

Takaaki Aoki ${ }^{1,2)}$ and Jiro Matsuo ${ }^{1)}$

1) Quantum Science and Engineering Center, Kyoto University

2) Collaborative Research Center for Advanced Quantum Beam Process Technology

\section{ABSTRACT}

Damage formation process by gas cluster impact was studied using molecular dynamics simulations. Ar gas clusters with various sizes (several tens to several thousand) and energies (several to several tens keV, totally) were irradiated on Si substrates. The relationship between mean damage depth, incident energy and cluster size was examined. The model of damage formation process and conditions to cause damage by cluster impact were discussed.

PACS: 6.40.-c Atomic and molecular clusters, 83.10.Rs (Computer simulations of molecular and particle dynamics)

KEYWORD: molecular dynamics, cluster ion beam, damage formation

\section{INTRODUCTION}

Gas cluster ion beam (GCIB) process is one of the promising techniques for high-speed and high-precision nano-machining [1]. The advantages of GCIB process are due to that a large cluster impact causes multiple collisions at the very shallow surface region of the target, which results in large motion of many surface atoms and enhancement of chemical reaction. As the development of nano-scale devices is more improved, damage formation during surface processes becomes more serious problem. As for GCIB process, it is expected that cluster size control is unique and effective technique to reduce process damage [2,3]. In this paper, damage formation process by cluster impact was examined using molecular dynamics simulations. The damage formation model was discussed through the investigation of damage profile and dependency on size and kinetic energy of incident clusters.

\section{SIMULATION MODEL}

In order to study collisional process between cluster and solid surface, molecular dynamics (MD) simulations of Ar clusters impacting on $\operatorname{Si}(001)$ surfaces were performed. MD simulation is one method of computer simulation to solve numerically the Newton's equation of motion for each atom in the system using difference equation technique, so MD can make it possible to trace the time evolution of coordinate and velocity for every atom with high resolution. In this simulation, the interatomic potentials were described with Stillinger and Weber model [4] for Si-Si. On the other 
hand, interactions for Ar-Si, and Ar-Ar were applied with only ZBL (Ziegler, Littmark, and Biersack) model [5], which consists of repulsive force. This implementation was based on the assumption that the attractive potentials between Ar-Si and Ar-Ar are small and negligible compared with kinetic energy of incident atoms and binding energy of $\mathrm{Si}$ atoms. In order to reduce calculation time and keep incident cluster stable, cut-off radii of $3.0 \AA$ and $3.7 \AA$ were applied to $\mathrm{Ar}-\mathrm{Si}$ and $\mathrm{Ar}-\mathrm{Ar}$ interactions, respectively. Ar clusters with spherical h.p.c. structure and size ranging from 10 to several thousands were prepared as the projectiles. Clusters were accelerated with various energies ranging several hundreds to several tens thousand $\mathrm{eV}$ and irradiated on $\mathrm{Si}(001)$ surface. The size of target was selected enough large to receive kinetic energy of incident cluster. For example, at the impact of Ar cluster with $20 \mathrm{keV}$, the target size was more than 2,000,000 and its dimensions were about $400 \AA \times 400 \AA \times 200 \AA$. The simulation time was 8 ps, which is enough long to observe the formation and recovery process of damage caused by cluster impact under above conditions.

\section{RESULTS and DISCUSSION}

Fig. 1 shows the snapshots of $\mathrm{Ar}_{2000}$ clusters with various incident energies impacting on $\mathrm{Si}(001)$ surface, 8ps after the impact. Large and small circles indicate Ar and Si atom, respectively, and displaced Si atoms are indicated as small gray circles. In this study, displaced atom is defined as an atom which is below $2.5 \AA$ from the surface level and which potential energy is above $0.2 \mathrm{eV}$ from bulk status [6]. When incident energy is $10 \mathrm{eV} /$ atom (totally $20 \mathrm{keV}$ ), the Ar cluster penetrates the $\mathrm{Si}$ surface. Large number of Si atoms are displaced spherically and a crater-like damage remains on the surface after the re-evaporation of incident Ar atoms. This crater-like damage is confirmed by in-situ STM observation of Si surface bombarded with Ar cluster ions [7]. As the incident energy decreases, penetration depth of cluster, depth and radius of crater decreases. When the incident energy is as low as $1 \mathrm{eV} /$ atom, as shown in Fig. 1, the cluster does not penetrate the surface but break-up on the surface. During the collisional process, some surface atoms are displaced, but the displaced length is small, so that, these displacements recover and no damage remains on the surface within several pico-seconds after the impact.

Fig. 2 shows the incident energy dependence of the mean depth of displaced Si atoms (written as small gray circles in Fig. 1) caused by impacts of Ar clusters with various sizes. The left viewgraph describes the dependency as the function of 'incident energy per atom,' while the right one gives as the function of 'total incident energy.' As shown in left viewgraph, the damage depth of $\mathrm{Ar}_{10}$ shows almost similar tendency to that of $\operatorname{Ar}_{1}$ (monomer). However, if the cluster size is more than 10 , the damage depth increases as the cluster size. From the viewpoint of the relationship between damage depth and incident energy, the damage depth increases proportionally to a cubic root of incident energy at the cluster sizes ranging from 20 to 200, while it increases non-linearly as the incident energy at the cluster size more than 1000 . 
On the other hand, the right viewgraph indicates that the impacts of small clusters with the size less than 200 show similar damage depth with each other. In this case, the cluster has enough large incident energy and penetrates into the target, which causes large number of collisions between cluster and surface atoms. During this multiple collision process, kinetic energy of the cluster is transferred not only in vertical but also in lateral directions. This spherical transmission of kinetic energy causes crater-like surface deformation shown in the right snapshot of Fig. 1. After the collisional process, the most of incident energy of cluster is transferred to the target to form damage, which means that the incident energy can be quantified as the volume of damaged region. If the aspect ratio of damaged region does not change depending on both cluster size and incident energy, it can be concluded that the damage depth does not depend on cluster size and is proportional a cubic root of the incident energy.

As for larger cluster impact, the penetration process into the target becomes important issue for damage formation. Even if total energy is as high as several $\mathrm{keV}$, incident energy-per-atom decreases as the cluster size increases. Finally, when cluster size is enough large, the cluster does not penetrate the surface of the target even if the proximity effect of cluster is considered. In this case, the cluster stays on the target and the most of incident energy of cluster is used to break itself (like the left snapshot in Fig. 1). From the left viewgraph in Fig. 2, a specific value of energy-per-atom can be found around $10 \mathrm{eV} /$ atom, where the damage depth does not obey $E^{1 / 3}$ law but shows rapid decline at lower incident energy. This specific incident energy-per-atom is independent on the cluster size and is similar to the energy to knock-on one target atom.

\section{SUMMARY}

Molecular dynamics simulations of Ar clusters impacting on Si substrates were performed in order to examine the relationship among damage formation, incident energy and cluster size. It was found that there is a specific incident energy, where a cluster penetrates into a target completely and cause multiple collisions. After the multiple collision process, a crater-like damage is formed on the surface. The dimensions of damaged layer were described as a simple function, a cubic root of the incident energy. These expectations from MD simulations will supports the design of GCIB processes for various applications of surface smoothing and high-speed sputtering of materials eliminating surface damage.

\section{ACKNOWLEDGEMENT}

This work is supported by New Energy and Industrial Technology Development Organization in JAPAN.

\section{REFERENCES}


[1] I. Yamada, J. Matsuo, Z. Insepov, T. Aoki, T. Seki and N. Toyoda, Nucl. Instr. and Meth., B 164-165 (2000) 944.

[2] T. Aoki, J. Matsuo and G. Takaoka, Nucl. Instr. Meth. B, 202 (2003) 278.

[3] T. Aoki, T. Seki, A. Nakai, J. Matsuo, G. Takaoka, Trans. Mat. Res. Soc. Japan, 28 (2003) 485

[4] F. H. Stillinger and T. A. Weber, Phys. Rev., B 31 (1985) 5632.

[5] J. F. Ziegler, J. P. Biersack and U. Littmark, The stopping and range of ions in solids (Pergamon Press, New York, U.S.A, 1985).

[6] M. J. Catula, T. diaz de la Rubia and G. H. Gilmer, Nucl. Instr. and Meth, B 196 (1995) 1.

[7] T. Seki, J. Matsuo, G. H. Takaoka and I. Yamada, Proc. of 16th international conference on the application of accelerators in research and industry, (AIP Conference Proceedings Vol. 576, 2001) 1003.

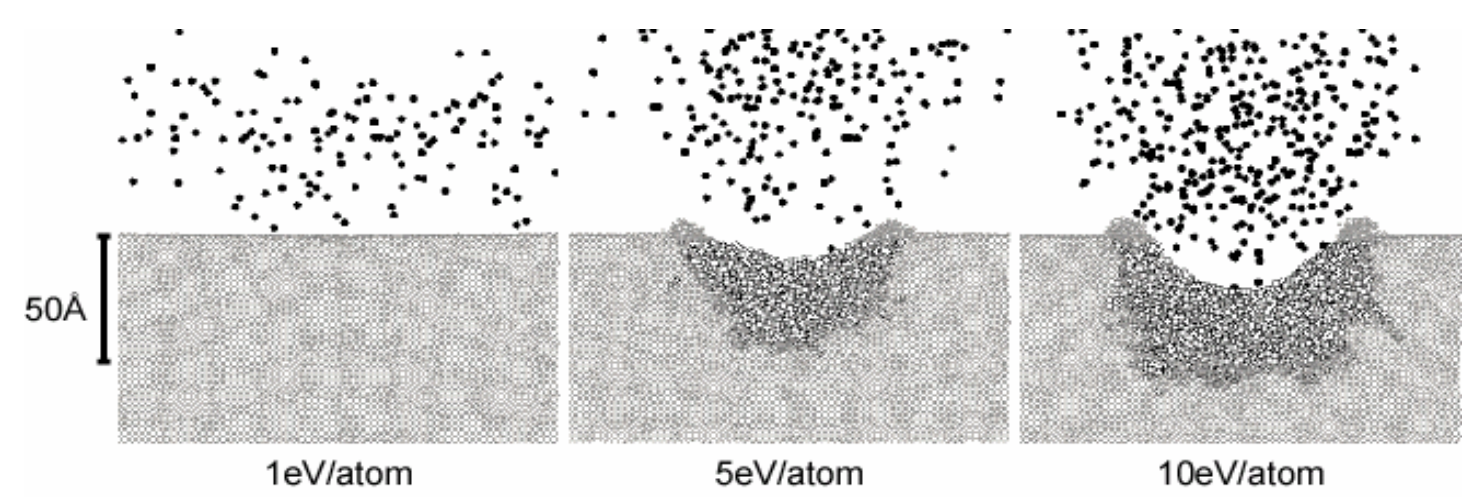

Figure 1: Snapshots of $\mathrm{Ar}_{2000}$ cluster with various incident energy-per-atom impacting on $\mathrm{Si}(100)$ surface, 8ps after impact.
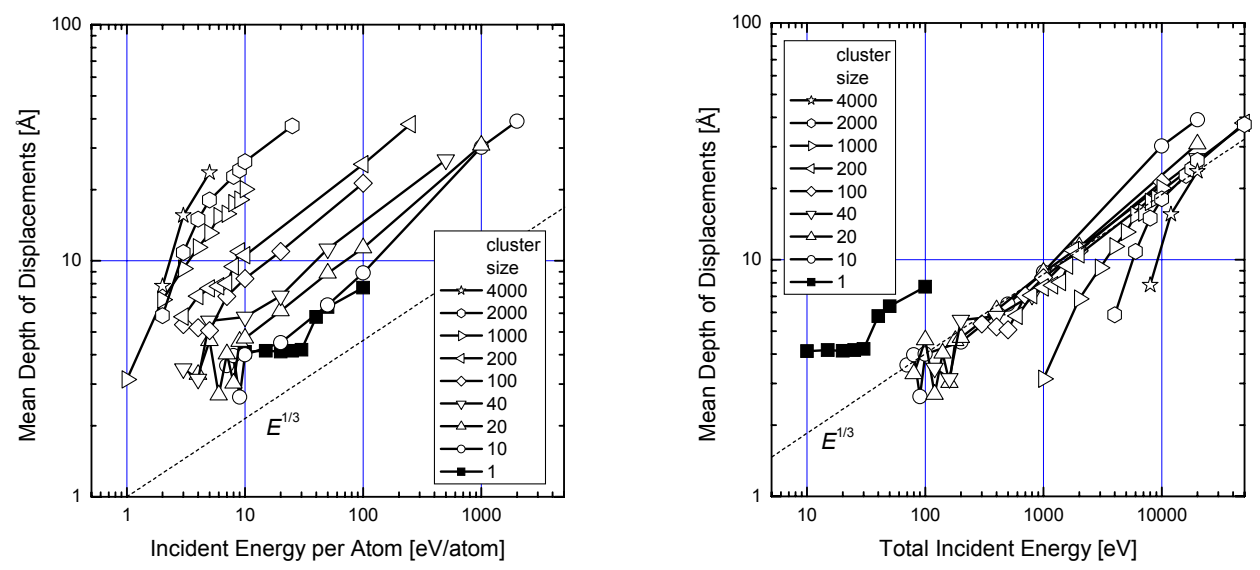

Figure 2: Incident energy dependence of the mean depth of displacements caused by various cluster impact. Left graph describes as the function of energy-per-atom and right one as total energy. 